\title{
Recours aux données pour révéler et sélectionner les adolescentes les plus vulnérables
}

Sarah Engebretsen

Population Council

Follow this and additional works at: https://knowledgecommons.popcouncil.org/departments_sbsr-pgy

Part of the Family, Life Course, and Society Commons, Gender and Sexuality Commons, International Public Health Commons, and the Women's Health Commons How does access to this work benefit you? Let us know!

\section{Recommended Citation}

Engebretsen, Sarah. 2012. "Recours aux données pour révéler et sélectionner les adolescentes les plus vulnérables," GIRLS FIRST! Perspectives de programmation centrée sur les filles. New York: Population Council. 
UNE PUBLICATION GIRLS FIRST!

RECOURS AUX

DONNÉES POUR

RÉVÉLER ET

SÉLECTIONNER

LES ADOLESCENTES

LES PLUS

VULNÉRABLES

PAR SARAH ENGEBRETSEN
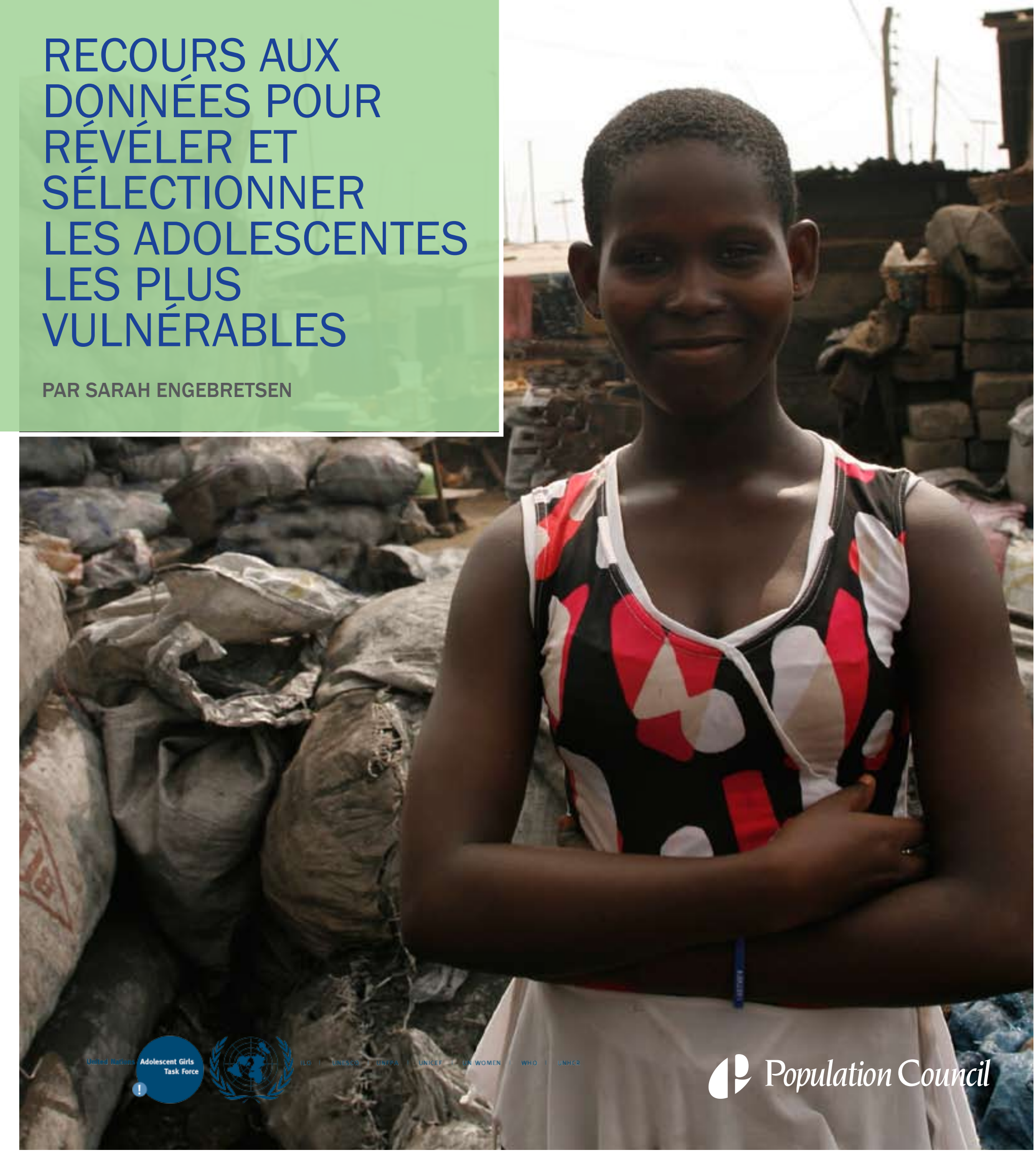


\section{GIRLS FIRST!}

\section{PERSPECTIVES DE PROGRAMMATION CENTRÉE SUR LES FILLES}

La recherche et les programmes consacrés aux adolescentes se concentrent traditionnellement sur la sexualité, la santé génésique et le comportement, négligeant la problématique socioéconomique plus large qui sous-tend leurs droits humains, leur développement général, leur santé et leur bienêtre. De plus, les efforts d'amélioration de la vie des filles mettent souvent en vedette ceux et celles qui dominent ou influencent leur vie (les parents, la belle-famille, les garçons, les hommes, les agresseurs), sans prêter aux intéressées elles-mêmes l'attention qu'elles méritent en soi.

GIRLS FIRST! Perspectives de programmation centrée sur les filles est une série de cinq comptes rendus thématiques, rédigés par les experts et expertes du Population Council. Ces dossiers présentent un instantané de la base de connaissances telle qu'elle se présente, à un moment donné, dans ce domaine en évolution permanente. Ils abordent les cinq priorités stratégiques définies dans la Déclaration conjointe des Nations Unies, "Intensifier l'action menée pour promouvoir les droits des adolescentes " (mars 2010), symbole de l'engagement collectif de sept organismes de l'ONU au soutien des gouvernements et de leurs partenaires pour la promotion de politiques et programmes essentiels au profit des adolescentes les plus difficiles à atteindre. Ces comptes rendus

1. explorent l'évolution prochaine de l'éducation des filles;

2. définissent les grandes lignes d'approches innovantes en vue de l'amélioration de la santé des filles;

3. recadrent l'approche de la violence à l'encontre des filles ;

4. décrivent les meilleurs moyens de cultiver les leaders féminines; et

5. expliquent de nouveaux modes inédits de collecte et d'exploitation des données relatives aux adolescentes.

Ils avancent des arguments novateurs en faveur de l'investissement dans les filles et mettent en valeur les pratiques prometteuses. Ils expriment un point de vue factuel progressiste quant aux allocations de ressources aptes à assurer le plus rapidement et le plus efficacement l'amélioration de la vie des filles.

Rédigés par des experts et expertes du Population Council, une organisation pionnière d'une recherche et programmation de pointe à l'intention des adolescentes vulnérables et marginalisées, ces rapports ont bénéficié, d'abord et surtout, de l'appui et du leadership de l'Équipe spéciale des Nations Unies pour les adolescentes. L'initiative a également reçu l'appui moral et financier de la Nike Foundation, de la Fondation des Nations Unies et de la David and Lucile Packard Foundation. Ces comptes rendus viennent s'ajouter à la guidance programmatique et aux " boîtes à outils " aujourd'hui disponibles ; ils offrent une ressource de référence essentielle à toute initiative d'élaboration de politiques et programmes efficaces et durables au profit des filles. Notre espoir est qu'ils inspireront de nouvelles approches utiles aux efforts de réalisation des droits des adolescentes marginalisées du monde.

Juillet 2012

Population Council et Équipe spéciale des Nations Unies pour les adolescentes

Avis

Ces comptes rendus sur la programmation pour adolescentes dans les domaines de l'éducation, de la santé, de la réduction de la violence, du leadership des filles et des données ont été préparés par le Population Council pour l'Équipe spéciale des Nations Unies pour les adolescentes, avec l'appui de la Nike Foundation, de la Fondation des Nations Unies et de la Packard Foundation.

Les opinions exprimées sont strictement celles des auteurs et ne reflètent pas nécessairement celles des partenaires donateurs.

(c) 2012 The Population Council, Inc. www.popcouncil.org 


\title{
RECOURS AUX DONNÉES POUR RÉVÉLER ET SÉLECTIONNER LES ADOLESCENTES LES PLUS VULNERABLES
}

\author{
PAR SARAH ENGEBRETSEN
}

PRINCIPES ET CONTEXTE DE LA POLITIQUE...................................................................................

Révéler les filles dans la vue d'ensemble « macro " et les lier aux objectifs du développement.............................

Recourir aux données pour identifier les filles dans lesquelles investir:

un choix stratégique, pratique et éthique

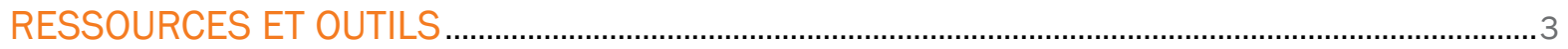

Les données désagrégées révèlent la diversité interne des adolescents et aident

à établir les priorités des investissements au profit de ceux qui en ont le plus besoin

La méthode des tables de mortalité identifie le début et l'étendue de la vulnérabilité

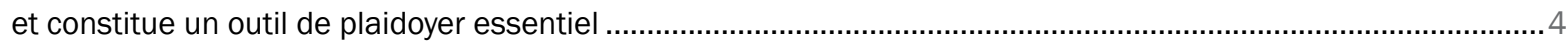

Les chiffres bruts révèlent les foyers de concentration de filles vulnérables en mal

de programmation ciblée

Évaluer la part féminine des ressources affectées aux jeunes :

l'outil "Exercice de couverture " .5

La technologie de la cartographie pour définir une communauté, identifier les filles

admises et déterminer celles qui sont atteintes .5

\section{APPLICATIONS SUR LE TERRAIN : QUATRE PROGRAMMES TIRANT \\ DYNAMIQUEMENT PARTI DES DONNÉES POUR CIBLER LES ADOLESCENTES}

LES PLUS VULNÉRABLES

Recours aux données désagrégées pour identifier les foyers régionaux

de vulnérabilité des filles au Bénin

Recours aux données désagrégées pour révéler le désavantage

des adolescentes du Ghana.

Exploration de l'équilibre entre le besoin et la couverture des adolescents

vulnérables au Burkina Faso. .8

Recours à la cartographie pour cibler et recruter les filles vulnérables, engager les filles au titre de chercheuses et engager la communauté au soutien d'un programme

de renforcement des compétences au Guatemala. 8 


\section{PRINCIPES GÉNÉRAUX ET CONTEXTE DE LA POLITIQUE}

Révéler les filles dans la vue d'ensemble " macro " et les lier aux objectifs du développement.

Il est crucial d'investir dans les adolescentes du monde en développement, où une proportion déjà importante et grandissante de la population tombe dans la catégorie des moins de 24 ans. Dans de nombreux pays en développement, le jeune âge au moment du mariage et de la procréation constitue une source considérable de croissance démographique à venir. De l'investissement dans les adolescentes dépendront largement l'avenir démographique, la protection des droits humains à la scolarité, à l'intégrité corporelle et au mariage légal et choisi, et le fardeau de la dépendance. Dans les pays où la fécondité est en baisse, il existe une possibilité de "dividende démographique " : un moment où une proportion grandissante de la population est à l'âge actif, donnant de l'élan au taux de croissance économique. En investissant dans les filles, on réduit leur fécondité tout en gonflant leur capacité productive, au profit des filles elles-mêmes, des familles, des communautés et, au sens large, de l'économie et de la société ${ }^{1}$.

Le monde a embrassé les Objectifs du Millénaire pour le développement, la Convention sur l'élimination de toutes les formes de discrimination à l'égard des femmes et la Convention internationale des droits de l'enfant. Ensemble, ces accords définissent un cadre de normes internationales des droits humains, de valeurs et d'action désirable en ce qui concerne les enfants et les adolescents. La réalisation de ces objectifs et de ces droits exige un investissement substantiel, concentré et réfléchi dans l'actif social, sanitaire et économique des jeunes des communautés les plus pauvres du monde. Les investissements consentis doivent l'être aux moments critiques de la jeune adolescence, quand le désavantage se consolide pour plus de 600 millions de filles dans le monde en développement. Atteindre cette population relève d'une stratégie essentielle de développement et de justice sociale, critique à la réalisation des Objectifs du Millénaire pour

\section{INVESTIR DANS LES ADOLESCENTES UNE STRATÉGIE D'ACCĖS AUX OBJECTIFS DU MILLÉ- NAIRE POUR LE DÉVELOPPEMENT}

Construire une forte base économique et renverser la pauvreté intergénérationnelle : Le contrôle accru du revenu par les femmes est plus rentable en termes de capital humain que celui d'un revenu comparable laissé aux hommes.

Assurer l'éducation primaire pour tous : Les filles des milieux ruraux en sont le plus privées.

Promouvoir l'égalité des sexes : La violence de genre et les pratiques traditionnelles néfastes entraînent de hauts niveaux de fécondité non désirée, de mortalité maternelle et de contraction du VIH.

Réduire la mortalité maternelle et la mortalité infantile qui en découle : Dans la plupart des cas, les mères primipares les plus jeunes courent le plus grand risque de mortalité maternelle.

Renverser la vague montante du VIH chez les jeunes : Les filles et les jeunes femmes, y compris les mères-enfants, représentent une part grandissante et disproportionnée des nouvelles infections. Le rapport d'incidence du VIH dans la tranche des 15 à 24 ans atteint 8 femmes pour 1 homme dans certains pays.

Freiner la croissance démographique galopante : Éliminer le mariage d'enfants et la maternité précoce qui y est associée renforce la productivité des filles et favorise une réduction synergique de la croissance démographique.

SOURCE : Bruce, Judith. "Reaching The Girls Left Behind: Targeting Adolescent Programming for Equity, Social Inclusion, Health, and Poverty Alleviation. " Préparé pour "Financing Gender Equality: A Commonwealth Perspective ", Commonwealth Women's Affairs Ministers' Meeting, Ouganda, juin 2007.

le développement ${ }^{2}$. (Voir l'encadré.)

Dans le monde en développement, les adolescents sont la proie de nombreuses violations de leurs droits humains, de la menace à leur intégrité corporelle au manque d'éducation et au mariage et à la procréation précoces. La réalisation de certains droits est différée (l'ouverture d'un compte d'épargne jusqu'à l'âge de 19 ans plutôt que de 16 ans dans un pays où le travail à temps plein est admis, par exemple). Les conséquences des infractions à certains droits peuvent être remédiables, même si le coût est élevé (interruption d'études), mais d'autres ne le sont pas (quand une personne subit des rapports sexuels forcés, contracte le VIH ou a un enfant alors qu'elle n'est elle-même pas encore sortie de l'enfance). Le fait est que la violation de tous droits humains menace le bien-être des adolescents, mais les responsables des politiques et des programmes doivent considérer ces catégories d'abus lors de l'allocation de leurs maigres ressources. Les plus jeunes adolescents courent souvent le plus grand risque d'abus. Les défis posés aux filles sont particulièrement lourds aux alentours de la puberté ${ }^{3}$, qu'il s'agisse de l'exclusion des espaces publics (et du manque de sécurité dans ceux accessibles), de la perte du réseau de pairs, de la déscolarisation, de la pression du mariage ou de liaisons comme stratégies de subsistance ou de l'intériorisation de normes de genre nuisibles, y compris celles relatives à la violence de genre. Cette problématique émergente affecte le développement psychosocial et la trajectoire suivie vers l'âge adulte ${ }^{4}$. Les données révèlent ces transitions et justifient la programmation ciblée.

Recourir aux données pour identifier les filles dans lesquelles investir : un choix stratégique, pratique et éthique.

II est indispensable d'investir dans les filles pour renforcer leur actif-protection. Mais pour effectuer ces investisse- 
ments, il faut d'abord " voir " ces filles. Les politiques de jeunesse actuelles et les données dont elles s'accompagnent bloquent la vue sur ces filles et traitent les jeunes comme un groupe homogène. Les compétences et le vécu des jeunes, même au sein d'une même communauté, peuvent cependant varier considérablement. Faute de reconnaître la variation des capacités et des débouchés offerts aux adolescents d'un sous-groupe à l'autre, ces politiques négligent souvent dans leurs allocations de ressources les adolescents vulnérables et difficiles d'accès. Leurs bénéficiaires sont généralement les jeunes déjà avantagés : urbains, plus âgés, de sexe masculin et scolarisés. Le problème se pose car ces populations disposent généralement d'un pouvoir socioéconomique supérieur et sont mieux placées pour profiter des ressources disponibles. Cela sans compter que les stratégies de recrutement ne s'attardent pas toujours sur la recherche des adolescents difficiles d'accès.

Il est essentiel de rendre les filles visibles si l'on veut que l'investissement soit ciblé sur les groupes qui présentent le plus grand risque de bilans non favorables. Tel est le principe de ce compte rendu : son but est d'identifier et cibler les adolescentes vulnérables et de façonner ainsi le contexte de la politique. II se veut guide de ressources et outils aptes à révéler la diversité interne des adolescents, à identifier les manifestations initiales et l'étendue de la vulnérabilité, à signaler les foyers de filles vulnérables, à évaluer dans les ressources affectées aux jeunes la part donnée aux filles et à rechercher les communautés et les filles vulnérables qui bénéficieraient d'une participation aux programmes. En conclusion, il présente quelques applications pratiques d'usage dynamique des données ${ }^{5}$. Si les Enquêtes démographiques et de santé (EDS) sont utilisées ici comme source de données, on notera que partout où des données de recensement sont disponibles, il est bon d'y prêter attention et d'étudier les populations cibles au niveau de la micro-analyse - même si les indicateurs disponibles sur les adolescents y sont plutôt limités ${ }^{6}$.

\section{RESSOURCES ET OUTILS}

Les données désagrégées révèlent la diversité interne des adolescents et aident à établir les priorités des investissements au profit de ceux qui en ont le plus besoin.

Les données désagrégées sont essentielles à la visibilité de l'hétérogénéité des sous-groupes d'adolescents. Ces données aident les décideurs, les gouvernements, les ONG et les organisations de plaidoyer à identifier les adolescents marginalisés, à prendre leurs décisions et à établir les priorités de l'investissement et, faute de données claires ou suffisantes, à motiver une recherche politique et programmatique complémentaire ciblée. À défaut d'une solide compréhension de la diversité interne de la population adolescente, ceux et celles les plus défavorisés risquent d'être laissés pour compte. Lors de l'examen des données, il importe de rechercher les écarts entre les différents groupes d'adolescentes, entre les milieux urbains et ruraux par exemple. Ces données peuvent aider à se faire une idée des différents types de conditions problématiques, ainsi que des périodes où ces conditions sont les plus intenses.

Les données de niveau national masquent souvent d'importantes divergences sous-nationales. II importe donc d'analyser les indicateurs au niveau sous-national (proportion des filles et jeunes femmes de 18 à 24 ans mariées avant l'âge de 15 ans dans chaque région d'un pays, par exemple) afin de révéler les " points chauds " ou foyers éventuels de sous-groupes vulnérables. Beaucoup d'ensembles de données permettent la représentation sous-nationale. Les responsables des politiques et de la programmation pourraient donc découvrir, par exemple, que le pourcentage de filles mariées avant l'âge de 15 ans dans un pays donné est inférieur à 15\%, mais qu'il s'élève à près de $50 \%$ dans une province particulière de ce pays.

Avec l'appui de l'UNFPA, le Population Council a élaboré des guides de données relatives aux adolescents, sur la base des données d'EDS, pour rendre visibles les groupes d'adolescents vulnérables. Ces guides mettent l'accent sur le début de l'adolescence, dans le but d'attirer l'attention sur les transitions à l'origine du désavantage'. Ils examinent ensuite le contexte de l'activité sexuelle des adolescents, en se concentrant tout particulièrement sur le mariage précoce, les normes de genre et la santé génésique ${ }^{8}$. Bien que les enquêtes en population générale ne collectent aucune information sur les adolescents de moins de 15 ans, Cynthia Lloyd, chercheuse au Population Council, a imaginé une méthode pionnière d'exploitation du cadre des ménages pour produire une image de la situation des 10 à 14 ans. Parmi les observations qui en découlent, il apparaît qu'une proportion importante d'adolescents est déscolarisée et ne vit pas avec ses parents. II en va notamment ainsi à Ouagadougou (Burkina Faso) pour $20 \%$ des filles âgées de 10 à 14 ans $^{9}$. Les guides de données relatives aux adolescents ont été conçus pour donner une image comparative au niveau des pays et d'un pays à l'autre. Ils dessinent la diversité interne des adolescents en décomposant les données autant que possible en fonction de l'âge, du sexe, des conditions de logement, de la scolarisation, de l'état matrimonial et du milieu de résidence actuel (urbain ou rural).

Les données incluses dans les guides, cartographiées de manière à indiquer les variations sous-nationales dans la plus large mesure possible, révèlent clairement les besoins profonds et indiquent les zones potentielles d'intervention. La liste ci-dessous présente quelques exemples types de données incluses dans les guides ${ }^{10}$ :

- Proportion des jeunes femmes de 20 à 24 mariées avant l'âge de 15 ans et avant l'âge de 18 ans.

- Proportion des filles de 10 à 14 ans vivant avec un parent ou sans parent.

- Proportion des filles d'âge scolaire ${ }^{11}$ déscolarisées.

- Proportion des filles scolarisées au niveau approprié pour leur âge ${ }^{12}$.

- Proportion des filles et jeunes femmes de 15 à 24 qui pensent que la violence à l'encontre des femmes est acceptable dans certaines circonstances.

- Proportion des filles qui ont déjà subi une forme de violence ${ }^{13}$. 
- Proportion des filles sexuellement actives.

- Différence d'âge entre les filles sexuellement actives et leur premier partenaire et partenaire actuel.

Ces indicateurs peuvent servir à confirmer les observations subjectives relatives aux adolescentes. Par exemple, les groupes d'action locale de Lusaka (Zambie) peuvent énumérer les défis auxquels les adolescentes se trouvent confrontées et chaque condition observable peut être confirmée - ou réfutée sur la base des données (Tableau 1).

La méthode des tables de mortalité identifie le début et l'étendue de la vulnérabilité et constitue un outil de plaidoyer essentiel.

Certaines données dressent un tableau de vulnérabilité à travers les cohortes d'âge, comme la proportion des filles et garçons de certains âges qui sont déscolarisés - cette mesure étant cependant moins utile quand une faible proportion d'élèves commence à aller à l'école à l'âge approprié. Si la proportion est plus élevée, les données reprises dans les guides permettent de cerner clairement les âges auxquels la déscolarisation commence et s'accélère. Par exemple, plus de $87 \%$ des filles des milieux ruraux du Guatemala sont scolarisées à l'âge de 10 ans, mais $27 \%$ seulement le sont à $17 \mathrm{ans}^{14}$. Le déclin rapide de la scolarisation des filles survient aux alentours de la puberté.

Le recours à la méthode des tables de mortalité ${ }^{15}$ est important partout où les données le permettent. Cette méthode révèle l'âge auquel un jalon particulier est franchi (plutôt que d'indiquer les moyennes sur l'ensemble des cohortes.) Ainsi, les données de l'EDS éthiopienne de 2010 indiquent que si 23\% seulement des filles de 15 à 19 ans de la région d'Amhara étaient mariées ou en union au moment de l'enquête, non moins de $56 \%$ des jeunes femmes mariées de 20 à 24 ans l'étaient déjà à l'âge de 18 ans $^{16}$-soit plus de deux fois la proportion des filles mariées. La méthode des tables de mortalité révèle la véritable ampleur de la problématique des filles. Les décideurs politiques et les responsables des programmes peuvent prendre cette information comme point de départ et procéder à reculons pour déterminer le moment optimal de l'investissement pour produire le plus grand impact sur les filles. Si une haute proportion des filles d'une certaine région étaient mariées avant l'âge de 15 ans, le processus de désinvestissement s'est vraisemblablement produit considérablement plus tôt. Si un accroissement des taux de déscolarisation était détecté parmi les filles de 13 ans, par exemple, on pourrait entreprendre une interven- tion auprès de celles de 10 à 12 ans pour éviter l'écueil.

Les chiffres bruts révèlent les foyers de concentration de filles vulnérables en mal de programmation ciblée

Les pourcentages n'offrent pas toujours le moyen le plus convaincant de présenter l'étendue d'un problème particulier : les chiffres bruts impressionnent souvent davantage les décideurs politiques. Accompagner les pourcentages de chiffres bruts peut dresser un tableau plus complet de la situation. Si les pourcentages donnent une idée générale de ce qui se passe en un endroit particulier, ils n'indiquent pas la taille du problème en ce qui concerne le nombre total d'adolescentes affectées par une certaine condition. En Éthiopie, par exemple, plus de $5 \%$ des filles de 10 à 14 ans ne sont pas scolarisées et sont séparées de leurs parents ${ }^{17}$. Si le pourcentage ne semble pas problématique en soi, il est frappant de constater qu'environ 281320 filles tombent dans cette catégorie en Éthiopie ${ }^{18}$. Bien que le pourcentage correspondant soit plus élevé au Mozambique (9\%), le nombre de filles concernées est moindre (130 960) étant donné la moindre taille de la population mozambicaine.

Les investissements à grande échelle donnent souvent la priorité aux

\section{TABLEAU 1 DE LA MESURE SUBJECTIVE À OBJECTIVE DU RISQUE POUR LES ADOLESCENTES DE LUSAKA (ZAMBIE)}

RISQUE OBSERVABLE COURU PAR LES ADOLESCENTES

Grossesse et maternité précoces

Violence physique

Isolement

Faible accès à l'éducation
MESURE OBJECTIVE DU RISQUE

21,4\% des filles de 15 à 19 ans ont déjà été enceintes ou eu un enfant.

40,5\% des filles de 15 à 24 ans ont subi une forme de violence physique.

$7,7 \%$ des filles de 10 à 14 ans sont déscolarisées et ne vivent ni avec leur mère, ni avec leur père.

29,7\% des filles de 7 à 18 ans ne sont pas scolarisées.l

SOURCE : Les données de ce tableau sont extraites de l'EDS de 2007. Un résumé des conclusions relatives aux adolescents a été publié [en anglais] dans The Adolescent Experience In-Depth: Using Data to Identify and Reach the Most Vulnerable Young People: Zambia 2007. 2009. New York : Population Council. 


\section{SCOLARISATION DES JEUNES DE 10 À 17 ANS}

AU GUATEMALA

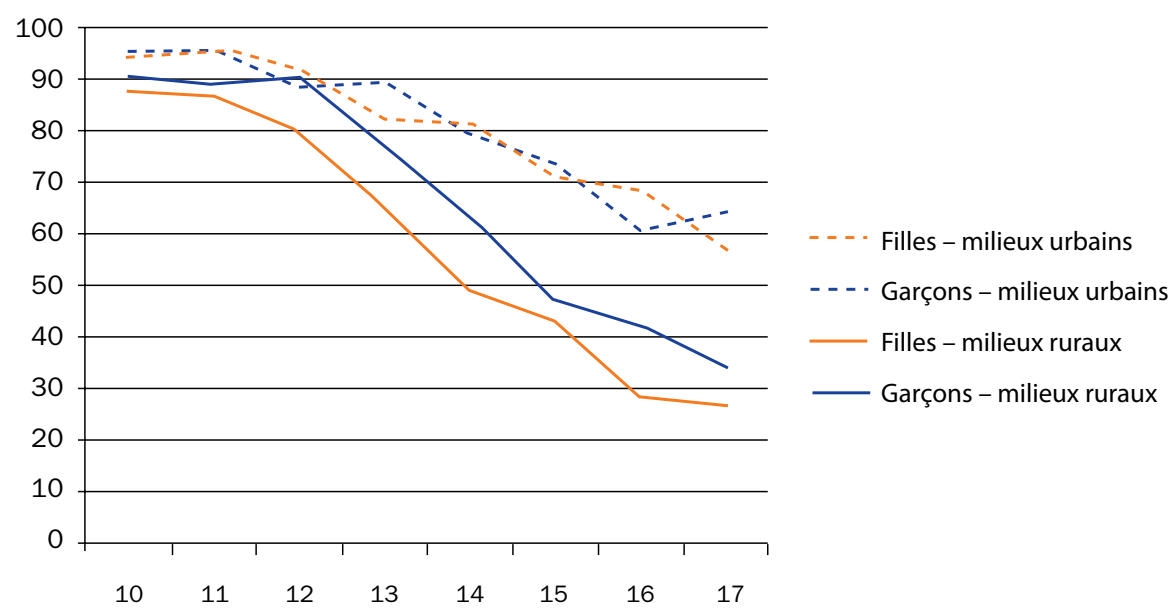

SOURCE : The Adolescent Experience In-Depth: Using Data to Identify and Reach the Most Vulnerable Young People: Guatemala 2002/2006. 2009. New York : Population Council. [En anglais.]

grands pays, mais une réallocation des ressources aux régions peut produire un nombre de bénéficiaires tout aussi impressionnant. Par exemple, s'il est admis que les taux de scolarisation sont généralement très faibles parmi les adolescentes d'Afrique occidentale francophone, le contre-argument est que ces pays ont des populations peu nombreuses et qu'ils ne méritent donc pas le même investissement que ceux plus peuplés. Un simple calcul révèle pourtant, sur la base des données de population adolescente des perspectives démographiques de l'ONU World Population Prospects 2008 et des données de scolarisation les plus récentes des Enquêtes démographiques et de santé, que le nombre de filles de 10 à 14 ans déscolarisées et qui font donc "fausse route " est pratiquement identique en Afrique occidentale francophone et au Nigeria. (Voir le Tableau 2.) Les chiffres absolus sont importants au processus de cartographie de l'ampleur d'un problème par rapport à celle de l'aide ciblée sur ce problème et à la décision des priorités de l'investissement.

\section{Évaluer la part féminine des} ressources affectées aux jeunes: l'outil "Exercice de couverture". Une fois révélée la nécessité de cibler les programmes sur les sous-groupes d'adolescents vulnérables, une analyse des programmes existants peut servir à évaluer l'usage féminin des ressources affectées aux jeunes. Le Population Council a créé un outil d'évaluation destiné à déterminer l'équité de l'accès aux programmes pour adolescents. Appelée " exercice de couverture ${ }^{19}$ ", cette ressource peut servir à déterminer les caractéristiques de base des participants ou non aux initiatives entreprises à l'intention des adolescents. II s'agit d'un outil d'évaluation économique, qui permet de profiler les bénéficiaires d'un programme particulier ou de plusieurs ${ }^{20}$. II y a souvent inversion des priorités.

En d'autres termes, ceux dotés dès le départ des plus grands avantages généralement les garçons plus âgés et scolarisés - bénéficient du plus grand nombre de ressources ; les moins favorisés au départ - les filles plus jeunes et déscolarisées - en bénéficient le moins. Un exercice de couverture réalisé par le Population Council et I'UNFPA ${ }^{21}$ révèle, au sein d'un échantillon de plus de 6000 adolescents fréquentant les services de jeunesse, une proportion de 63\% de jeunes de plus de 20 ans, par rapport à 7\% seulement de bénéficiaires âgés de 10 à 14 ans $^{21}$, indiquant que le groupe le plus jeune et sans doute le plus vulnérable est à peine atteint. D'après les résultats d'une analyse similaire réalisée en Mauritanie, 83\% des bénéficiaires des programmes pour les jeunes sont de sexe masculin ${ }^{22}$. Les résultats de l'exercice de couverture peuvent aider les organisations à rediriger les ressources vers ceux et celles qui en ont le plus besoin et à définir des approches mieux ciblées pour les atteindre.

La technologie de la cartographie pour définir une communauté, identifier les filles admises et déterminer celles qui sont atteintes.

La technologie de la cartographie aide à collecter une information importante à la programmation. Pus précisément, la collecte de données mobiles peut servir à définir une communauté, à identifier les filles admises et à déterminer les personnes atteintes par un programme donné. Un système de localisation GPS peut relever les coordonnées de latitude et de longitude de l'emplacement d'un programme, pour les charger aisément dans une application telle que Google Earth et générer des cartes aériennes de la couverture du programme. Ces cartes peuvent représenter l'emplacement d'un programme destiné exclusivement aux filles et fixer l'attention sur la zone d'influence ou communauté cible dont proviennent les bénéficiaires. L'établissement de la zone d'influence d'un programme donne une idée réaliste de la taille et des limites d'une communauté donnée et facilite la sélection d'emplacements appropriés pour le programme. La cartographie peut aussi indiquer les lieux de présence ou non de programmes et permettent la différenciation des programmes actifs sur le terrain de ceux qui défendent la cause des adolescentes sans toutefois transférer

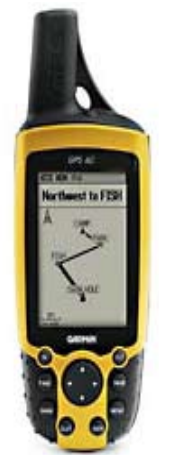

Appareil GPS de ressources.

La technologie de la cartographie peut aussi servir à identifier les ménages comptant des adolescentes vulnérables potentiellement admises à participer à un programme donné. En allant de 
TABLEAU 2 CALCULS D'ESTIMATION DU NOMBRE DE FILLES DE 10 À 14 ANS DÉSCOLARISÉES

\begin{tabular}{|c|c|c|c|c|}
\hline & & $\begin{array}{l}\text { \% POPULATION } \\
\text { FÉMININE }\end{array}$ & & $\begin{array}{l}\text { ESTIMATION } \\
\text { DU NOMBRE }\end{array}$ \\
\hline POPULATION & & DE 10 À 14 & & DE FILLES \\
\hline $\begin{array}{l}\text { FÉMININE DE } \\
10 \AA ̀ \text { A } 14 \text { ANS }\end{array}$ & $\mathbf{x}$ & $\begin{array}{l}\text { ANS DÉSCOLARISÉE } \\
\text { (ANNÉE) }\end{array}$ & $=$ & $\begin{array}{l}\text { DE } 10 \text { À } 14 \text { ANS } \\
\text { DÉSCOLARISÉES }\end{array}$ \\
\hline
\end{tabular}

Afrique occidentale francophone

Bénin

553369

$37,6(2006)$

208067

Burkina Faso

987093

$71,1(2003)$

701823

Guinée

617935

$48,6(2005)$

300316

Mali

824359

$57,8(2006)$

476480

Niger

997839

70,2 (2006)

700483

Sénégal

805530

$43,5(2005)$

350406

2737575

Nigeria

9488199

29,7 (2003)

2817995

SOURCE : Tabulations par Sarah Engebretsen sur la base des guides de données Adolescent Experience In-Depth Data Guides et des projections de I'ONU.

porte à porte avec un appareil GPS, on peut collecter l'âge et le sexe de tous les membres de chaque ménage. Les coordonnées du GPS permettent d'identifier l'endroit exact où vivent les adolescentes. II s'agit là d'une information importante pour une population souvent invisible dans l'espace public. Elle aide les planificateurs des programmes à déterminer la proportion seuil de filles requise dans la communauté (une recommandation générale préconise environ 30\%) pour intensifier le capital social des participantes. Cette information révèle en outre les filles qui sont atteintes et celles qui ne le sont pas, aspect particulièrement important quand on sait que ce sont souvent les plus vulnérables qui s'absentent des programmes ou présentent les plus hauts taux d'abandon.

\section{APPLICATIONS SUR LE TERRAIN : QUATRE PROGRAMMES TIRANT DYNAMIQUEMENT PARTI DES DONNÉES POUR CI- BLER LES ADOLESCENTES LES PLUS VULNÉRABLES}

Recours aux données désagrégées pour identifier les foyers régionaux de vulnérabilité des filles au Bénin.

L'image des adolescentes du Bénin est celle d'une vulnérabilité généralisée. Une équipe de I'UNFPA Bénin et d'OSV Jordan (une ONG) a participé à un atelier mené sous la conduite du Population Council et financé par l'UNFPA sur la programmation et le renforcement de capacités des adolescentes. Le but était d'apprendre à utiliser les données pour identifier les sous-groupes de jeunes

\section{$68,6 \% \times 41113=28204$}

Pourcentage de filles dans la catégorie vulnérable

$x$ Nombre total de filles comprises dans la tranche d'âge

$=$ Nombre total de filles vulnérables dans la tranche d'âge donnée

vulnérables aux fins de la programmation. Ces partenaires ont eu recours aux données d'EDS présentées dans le guide de données 2006 sur les adolescents du Bénin pour estimer le nombre de filles comprises dans certains foyers de vulnérabilité : les filles déscolarisées, séparées de leurs parents, analphabètes et mariées ou en union. Supposant que les proportions listées dans le guide des données 2006 demeureraient constantes au fil du temps, ils ont multiplié ces pourcentages par les prédictions 2010 du nombre total de filles comprises dans chaque tranche d'âge ${ }^{23}$ pour quantifier les nombres absolus de villes vulnérables. Ainsi, en 2006, par exemple, 69\% des filles de 10 à 14 ans de la région d'Alibori étaient déscolarisées. Si l'on considère cette proportion constante jusqu'en 2010, Iorsqu'Alibori comptait un total de 41113 filles dans la tranche de 10 à 14 ans, 28204 filles de 10 à 14 ans étaient alors déscolarisées dans la région. (Voir l'encadré ci-après et le Tableau 3.)

Ces données soulignent la multitude et l'ampleur des problèmes auxquelles les filles se trouvent confrontées dans la région d'Alibori. 
Les partenaires à cet exercice travaillent actuellement à la conception d'une intervention où la priorité de l'investissement serait accordée à ces filles avant leur transition critique vers la vulnérabilité. Pour plus d'information, s'adresser à Josephat Avoce (osvjordan@yahoo.fr).
Recours aux données désagrégées pour révéler le désavantage des adolescentes du Ghana.

La Women's Initiative for Self-Empowerment (WISE, initiative d'autonomisation des femmes par les femmes) a étudié le guide des données relatives aux adolescents du Ghana dans le but de mieux comprendre la situation défavorisée des filles et de concevoir une intervention en conséquence. Ce groupe a participé à deux ateliers du Population Council sur la programmation et le renforcement de capacités au bénéfice des adolescentes. Durant le second atelier, il a présenté un aperçu de la situation ghanéenne sur la

\section{ÉTABLIR DES REPĖRES DE SUCCÈS APPROPRIÉS POUR LES GROUPES DE FILLES VISÉS}

Une fois la population cible sélectionnée, les repères de succès doivent être établis. Les planificateurs doivent bien comprendre où se trouvent les filles en début de programme pour concevoir une intervention appropriée. Ils doivent définir les indicateurs au niveau de la fille et les mesurer avant et après l'intervention. Le changement doit être mesuré au niveau de la fille plutôt qu'à celui des gardiens présents dans sa viea.

Les indicateurs au niveau de la fille constituent d'importants repères de succès et il n'est pas rare de vouloir mesurer le bien-être des adolescents selon un indice. Or le problème posé par cette méthode est qu'elle rassemble trois conditions distinctes qui devraient plutôt être considérées séparément, en tout cas aux fins de la programmation. L'image du succès et les objectifs poursuivis pour la population de filles ciblée tombent dans trois grandes catégories :

- Les conditions qui doivent être réduites à zéro, notamment le mariage précoce, la contraction du VIH ou la maternité alors que la fille est elle-même encore enfant.

- Les conditions à maximiser, comme l'accès à la scolarisation suffisante recommandée.

- Les conditions ayant trait à la part équitable des ressources disponibles, comme l'accès à l'emploi au moment désiré.

aUne liste complète d'indicateurs mesurés au niveau des filles est présentée au chapitre 13 de l'ouvrage Conception d'un programme centré sur les filles : Boîte à outils pour l'élaboration, le renforcement et l'expansion de programmes destinés aux adolescentes. 2010. New York: Population Council. Disponible sur http://www.popcouncil.org/ pdfs/2011PGY_AdolGirlToolkit_fr.pdf.

\section{TABLEAU 3 ESTIMATION DES NOMBRES DE FILLES COMPRISES DANS LES CATÉGORIES VULNÉRABLES AU BÉNIN}

\begin{tabular}{|c|c|c|c|c|c|}
\hline RÉGION & $\begin{array}{c}\text { FILLES DE } \\
10 \text { À } 14 \text { ANS } \\
\text { DÉSCOLARISÉES }\end{array}$ & $\begin{array}{c}\text { FILLES DE } \\
10 \text { À } 14 \text { ANS } \\
\text { DÉSCOLARISÉES } \\
\text { ET SÉPARÉES DE } \\
\text { LEURS PARENTS }\end{array}$ & $\begin{array}{c}\text { FILLES DE } \\
15 \text { À } 19 \text { ANS } \\
\text { DÉSCOLARISÉES }\end{array}$ & $\begin{array}{c}\text { FILLES DE } \\
15 \text { À } 19 \text { ANS } \\
\text { ANALPHABÈTES }\end{array}$ & $\begin{array}{l}\text { FILLES DE } \\
15 \text { À } 19 \text { ANS } \\
\text { MARIÉES } \\
\text { OU EN UNION }\end{array}$ \\
\hline Alibori & 28208 & 2302 & 35137 & 34251 & 22269 \\
\hline Atacora & 21249 & 425 & 27497 & 25641 & 13142 \\
\hline Atlantique & 22731 & 762 & 30763 & 24310 & 8511 \\
\hline Borgou & 24676 & 834 & 32643 & 21425 & 20150 \\
\hline Collines & 15940 & 93 & 22273 & 18063 & 7477 \\
\hline Couffo & 13993 & 183 & 16908 & 14537 & 4393 \\
\hline Donga & 8957 & 397 & 13340 & 9454 & 7205 \\
\hline Littoral & 19078 & 1192 & 32347 & 18493 & 2606 \\
\hline Mono & 6427 & 152 & 9325 & 7613 & 4258 \\
\hline Ouémé & 20026 & 646 & 30345 & 22857 & 5855 \\
\hline Plateau & 16438 & 637 & 18846 & 17380 & 6272 \\
\hline Zou & 19241 & 658 & 25065 & 21087 & 9570 \\
\hline National & 216964 & 8281 & 294489 & 235111 & 111708 \\
\hline
\end{tabular}

REMARQUE : La présentation complète des calculs du Bénin concernant les filles vulnérables s'intitule Aperçu des groupes cibles Bénin et peut être consultée sur <http://www.popcouncil.org/mediacenter/events/2010AdolGirlsProgrammingBurkina.asp>. 
base des faits et des chiffres du guide de données. Cette présentation a révélé clairement les taux de scolarisation en baisse au fil de la progression en âge des adolescentes et la hausse du pourcentage des filles de 15 à 19 ans vivant en milieu urbain par rapport à leurs cadettes de 10 à 14 ans. Le groupe fait remarquer que les tendances de migration observées sont typiques aux Ghanéennes et que la migration s'effectue généralement du nord vers les grandes villes d'Accra et de Kumasi, notamment. La présentation a également souligné le nombre de filles séparées de leurs parents et déscolarisées, et leur vulnérabilité ultérieure à l'exploitation. Sur la base de ces données, WISE a conçu une intervention visant les filles de 10 à 20 ans déscolarisées des milieux urbains employées comme porteuses sur les marchés locaux. Pour plus d'information, s'adresser à Adwoa Bame (abame@wise-up.org).

\section{Exploration de l'équilibre entre le besoin et la couverture des adoles- cents vulnérables au Burkina Faso.}

Voir les adolescents défavorisés et décider des approches programmatiques pertinentes exigent le recours à plusieurs sources de données. Le Population Council a examiné les données d'EDS du Burkina Faso complétées de celles d'une enquête nationale menée en 2004 auprès des adolescents par le Guttmacher Institute (questions posées à différents groupes spécifiques d'adolescents et consultation des adolescentes par le biais d'entretiens en profondeur). Ces données illustrent les besoins des adolescentes, concernant en particulier l'information de santé sexuelle et génésique. Afin de déterminer si celles présentant le plus grand besoin recevaient la priorité des initiatives de jeunesse existantes, le Population Council, avec l'appui de I'UNFPA, a réalisé en 2005 un exercice de couverture auprès de 20 organisations vouées aux jeunes.

Les efforts conjugués du Population Council, de I'UNFPA et du Guttmacher Institute révèlent combien les adolescents qui en ont le plus besoin sont les moins représentés dans les programmes proposés aux jeunes. Les cadettes et les adolescentes récemment mariées ne disposent notamment pas d'une

\section{COLLECTE DE DONNÉES SENSIBLES AUPRÈS DES ADOLESCENTS : L'AUTO-ENTRETIEN AUDIO ASSISTÉ PAR ORDINATEUR}

II existe différentes technologies utiles à la découverte de vastes groupes d'adolescents souvent négligés. Les chercheurs du Population Council ont mis au point le logiciel d'auto-entretien audio assisté par ordinateur (ACASI), dans le but de réduire les réponses inexactes aux questions d'enquête sensibles potentiellement embarrassantes ou gênantes lorsqu'elles sont posées dans le cadre d'un entretien en tête à tête. Avec l'outil ACASI, les répondants entendent les questions enregistrées et y répondent sur un appareil portable. On respecte ainsi le besoin de confidentialité et on obtient des réponses plus franches, objectif particulièrement important dans le cas des adolescentes du monde en développement où le comportement sexuel est souvent cause d'opprobre et la liberté d'expression féminine, souvent limitée. Les évaluations de la technologie indiquent une plus grande propension à déclarer les comportements sensibles lors d'entretiens informatisés que d'entrevues en tête à tête. Même parmi les populations à faible scolarisation ou haut niveau d'analphabétisme, ces outils sont bien acceptés et faciles à utiliser ${ }^{\mathrm{a}, \mathrm{b}}$.

aPour plus d'information sur l'outil ACASI, voir <http://www.popcouncil.org/projects/246_ ACASI/asp>. ' 'Une information plus complète sur les applications de l'outil ACASI auprès des jeunes est présentée [en anglais] dans "Obtaining more accurate and reliable information from adolescents regarding STI/HIV risk behaviors. "Promoting Healthy, Safe, and Productive Transitions to Adulthood. Barbara Mensch et Paul C. Hewett. 2007. New York : Population Council.

information suffisante et sont sous-représentées dans les programmes. Sept pour cent seulement des adolescents atteints par les initiatives à l'intention des jeunes sont âgés de 10 à 14 ans, par exemple, alors que les filles de 12 à 14 ans sont moins informées sur la santé sexuelle et génésique que celles de 15 à 19 ans (15\% par rapport à 31\% en ce qui concerne la connaissance des IST ; $22 \%$ par rapport à $47 \%$ pour ce qui est de la connaissance d'un endroit où se procurer des contraceptifs). De même, un quart seulement des adolescents atteints par les pairs éducateurs ne sont pas scolarisés, alors que la majorité des adolescents du Burkina Faso ne l'ont jamais été ${ }^{24}$. Ces constatations établissent clairement la nécessité d'un meilleur ciblage des ressources sur les plus vulnérables. Elles se sont avérées utiles à la conception d'une intervention destinée aux filles sujettes au risque du mariage précoce et à celles récemment mariées des régions rurales du Burkina Faso. Ce projet pilote, sous la conduite du Population Council, de I'UNFPA, de I'UNICEF et du ministère de l'Action sociale, est axé sur l'amélioration de la connaissance concernant la santé sexuelle et génésique et l'accès aux services. Pour plus d'information, s'adresser à Gisele Kabore (gkabore@ popcouncil.org), directrice nationale du Population Council au Burkina Faso.

Recours à la cartographie pour cibler et recruter les filles vulnérables, engager les filles au titre de chercheuses et engager la communauté au soutien d'un programme de renforcement des compétences au Guatemala.

Les collègues du Population Council responsables du programme Abriendo Oportunidades au Guatemala ont inauguré l'usage de la cartographie en tant qu'outil de ciblage et de recrutement des filles vulnérables, d'engagement des filles au titre de chercheuses et d'engagement de la communauté au soutien d'un programme de renforcement des compétences. Abriendo Oportunidades est un programme conçu à l'intention des filles mayas de 8 à 17 ans qui se trouvent confrontées aux problèmes du mariage précoce, d'une scolarisation limitée, d'une maternité fréquente, de l'isolement social et de la pauvreté chronique. Le personnel du programme a cartographié 
les ménages aux fins du ciblage et du recrutement des filles vulnérables invitées à participer. Tandis que le programme continue à s'étendre vers de nouvelles communautés, ses responsables recourent à la technologie GIS pour cartographier les limites de chaque communauté. À l'image de la plupart des communautés les plus pauvres et souvent informelles du monde, celles-ci ne sont pas formellement démarquées par des limites administratives officielles. Les responsables du programme ont aussi recours à la cartographie pour documenter le nombre de filles vivant dans chaque foyer, les ménages des leaders de la communauté et l'emplacement des ressources naturelles. Ces données servent ensuite à déterminer le potentiel d'expansion du programme vers de nouvelles communautés. Le programme a d'ailleurs engagé les filles dans le processus de collecte des données cartographiées. La technologie autonomise les filles, de même que l'occasion qui leur est donnée de découvrir ainsi leurs communautés. L'élaboration et le partage des cartes avec la communauté constituent une activité précieuse du programme. Elle favorise l'approbation locale des programmes de filles et suscite l'attention et l'engagement de tous les intéressés. Mieux encore, le processus de cartographie aide à poser les filles en véritables leaders de la communauté. Pour plus d'information, s'adresser au consultant Angel del Valle (adelvalle@popcouncil.org).

\section{NOTES ET RÉFÉRENCES}

1 Bruce, Judith. " Reaching the girls left behind: Targeting adolescent programming for equity, social inclusion, health, and poverty alleviation. " 2007. Dossier préparé pour la rencontre Financing Gender Equality: A Commonwealth Perspective, Commonwealth Women's Affairs Ministers' Meeting, Ouganda, 2007.

2 Ibid.

3 Les besoins des très jeunes adolescents sont cernés dans un document-guide et boîte à outils publié par le Population Council et I'UNFPA. Cherchant à combler les lacunes de la recherche et de l'attention concernant les jeunes de 10 à 14 ans, cet ouvrage propose une compilation de nouvelles approches de la collecte de données, d'outils et de méthodologies. Les méthodologies décrites dans le guide sont principalement utiles à l'identification des très jeunes adolescents les plus vulnérables, de leurs besoins et à la détermination de l'existence ou non de programmes qui les atteignent déjà. " Investing When It Counts: Generating the Evidence Base for Policies and Programmes for Very Young Adolescents. " <http://www.popcouncil.org/ pdfs/InvestingWhenltCounts.pdf> ou http:// www.unfpa.org/public/pid/363 [en anglais].

4 Bruce, Judith et Amy Joyce. 2006. "The failed reach of current schooling, child health, youth-serving, and livelihoods programs for girls living in the path of HIV ", chapitre 3 dans Judith Bruce, Nicole Haberland, et al. (eds.), The Girls Left Behind. Policy paper. New York: Population Council. [En anglais.]

5 Ce document se concentre sur la manière d'utiliser les données pour détecter les populations cibles et concevoir des programmes appropriés à leur intention. Pour plus d'information sur le suivi et l'évaluation des programmes pour adolescentes, voir les chapitres 12 et 13 de Conception d'un programme centré sur les filles : Boîte à outils pour l'élaboration, le renforcement et l'expansion de programmes destinés aux adolescentes. 2010. New York : Population Council.

6 Avec l'appui de I'UNFPA, le Population Council travaille à l'élaboration de directives de recensement d'information ciblée sur les adolescents. Ces directives, qui seront appliquées au Libéria dans le cadre d'une étude de cas, seront disponibles en 2012.

7 Pour une information sur les questions d'éthique et la protection des droits des enfants dans la collecte de données, voir Protecting children's rights in the collection of health and welfare data. http://www. popcouncil.org/publications/abstract. asp?ReflD=4536. [En anglais.]

8 Ces guides de données ont été élaborés pour une cinquantaine de pays. Pour une liste des guides disponibles et plus d'information, voir <http://www.popcouncil.org/publications/serialsbriefs/AdolExplnDepth.asp.> et $<$ http://www.unfpa.org/youth/dhs_adolescent_guides.html>.

9 Étude approfondie sur les adolescents : Exploiter les données pour identifier et atteindre les jeunes les plus vulnérables: Burkina Faso 2003. 2009. New York : Population Council.

10 La majorité des indicateurs repris sur cette liste figure dans l'EDS et est incluse dans la série The Adolescent Experience In-Depth: Using Data to Identify and Reach the Most Vulnerable Young People, produit d'un effort de collaboration entre le Population Council et I'UNFPA. Pour plus d'information et un lien d'accès aux guides de données relatives aux adolescents, voir <http://www.popcouncil. org/publications/serialsbriefs/AdolExplnDepth.asp> et <http://www.unfpa.org/youth/ dhs_adolescent_guides.html>.

11 La définition de l'âge de la scolarisation est spécifique à chaque pays et provient de I'UNESCO.
12 Cet indicateur figure dans l'EDS. Les analyses spécifiques aux pays sont résumées dans Cynthia B. Lloyd et Juliet Young, New Lessons: The Power of Educating Adolescent Girls. 2009. New York : Population Council. [En anglais.]

13 Certaines enquêtes EDS (pas toutes) comprennent des questions sur le vécu de la violence. Pour une liste des enquêtes couvrant la violence, voir http://www.measuredhs. com/topics/gender/dv_surveys.cfm\#1 [en anglais].

14 The Adolescent Experience In-Depth: Using Data to Identify and Reach the Most Vulnerable Young People: Guatemala 2002/2006. 2009. New York : Population Council.

15 Une description complète de la méthode des tables de mortalité est proposée dans Yaukey, Anderton et Lundquist. Demography: The Study of Human Population. 3rd Edition. 2007. Illinois: Waveland Press. [En anglais.]

16 Enquête démographique et de santé 2011, Éthiopie.

17 Ibid.

18 Calculs basés sur la multiplication du pourcentage de filles de 10 à 14 ans déscolarisées en Éthiopie (5,2\%) d'après l'EDS 2011 par la population féminine éthiopienne de 10 à 14 ans, soit 5410000 d'après les données de l'ONU dans World Population Prospects, 2008 Revision. Un calcul similaire a été effectué pour le Mozambique, par multiplication du pourcentage de filles de 10 à 14 ans déscolarisées au Mozambique (9\%) d'après l'EDS 2003 par la population féminine mozambicaine de 10 à 14 ans, soit 1455000 d'après les données de l'ONU dans World Population Prospects, 2008 Revision.

19 Un guide de conduite d'exercice de couverture est proposé sur www.popcouncil.org/pdfs/ CoverageExerciseGuide.pdf [en anglais].

20 Pour plus d'information sur l'exercice de couverture, voir www.popcouncil.org/pdfs/ TABriefs/28_AssessAccess.pdf [en anglais].

21 Les résultats de l'exercice de couverture sont résumés dans http://www.popcouncil.org/ pdfs/TABriefs/28_AssessAccess.pdf [en anglais].

22 lbid.

23 Bénin Projections Départementales : 2002-2030. Institut National de la statistique et de l'analyse économique. 2008.

24 Pour plus d'information sur l'étude de cas du Burkina Faso concernant l'accès aux adolescents présentant le plus grand besoin et l'effort conjugué du Population Council, de I'UNFPA et du Guttmacher Institute, voir <http://www.popcouncil.org/mediacenter/ events/2010AdolGirlsProgrammingBurkina. asp $>$.. 


\section{(2) Population Council}

Le Population Council s'attelle aux questions cruciales de la santé et du développement - qu'il s'agisse d'endiguer la progression du VIH, d'améliorer la santé reproductive ou d'offrir aux jeunes la promesse d'une vie satisfaisante et productive. Par ses travaux de recherche en biomédecine, en sciences sociales et en santé publique dans 50 pays, il s'efforce d'apporter avec ses partenaires des solutions qui conduisent à des politiques, des programmes et des technologies plus efficaces, pour améliorer la vie partout dans le monde. Fondé en 1952, le Population Council est une organisation non gouvernementale à but non lucratif. II siège à New York sous la conduite d'un conseil d'administration international. 